\title{
Double sperm cloning (DSC) is a promising strategy in mammalian genetic engineering and stem cell research
}

\author{
Zhi-ping Zhang ${ }^{\dagger}$, Jun-tao Zhang ${ }^{\dagger}$, Shu-cheng Huang $\mathbb{D}$, Xiu-yuan He and Li-xin Deng ${ }^{*}$
}

\begin{abstract}
Embryonic stem cells (ESCS) derived from somatic cell nuclear transfer (SCNT) and induced pluripotent stem cells (iPSCs) are promising tools for meeting the personalized requirements of regenerative medicine. However, some obstacles need to be overcome before clinical trials can be undertaken. First, donor cells vary, and the reprogramming procedures are diverse, so standardization is a great obstacle regarding SCNT and iPSCs. Second, somatic cells derived from a patient may carry mitochondrial DNA mutations and exhibit telomere instability with aging or disease, and SCNT-ESCs and iPSCs retain the epigenetic memory or epigenetic modification errors. Third, reprogramming efficiency has remained low. Therefore, in addition to improving their success rate, other alternatives for producing ESCS should be explored. Producing androgenetic diploid embryos could be an outstanding strategy; androgenic diploid embryos are produced through double sperm cloning (DSC), in which two capacitated sperms (XY or XX, sorted by flow cytometer) are injected into a denucleated oocyte by intracytoplasmic sperm injection (ICSI) to reconstruct embryo and derive DSC-ESCs. This process could avoid some potential issues, such as mitochondrial interference, telomere shortening, and somatic epigenetic memory, all of which accompany somatic donor cells. Oocytes are naturally activated by sperm, which is unlike the artificial activation that occurs in SCNT. The procedure is simple and practical and can be easily standardized. In addition, DSC-ESCs can overcome ethical concerns and resolve immunological response matching with sperm providers. Certainly, some challenges must be faced regarding imprinted genes, epigenetics, X chromosome inactivation, and dosage compensation. In mice, DSC-ESCs have been produced and have shown excellent differentiation ability. Therefore, the many advantages of DSC make the study of this process worthwhile for regenerative medicine and animal breeding.
\end{abstract}

Keywords: Embryonic stem cell, Double sperm cloning, Regenerative medicine, Reprogramming

\section{Introduction}

Stem cells represent a potential option for the treatment of some major diseases, such as cancer and degenerative diseases. Embryonic stem cells (ESCs) are the best option, representing the "gold standard"; they are obtained from the early mammalian embryo or IVF embryo and

\footnotetext{
* Correspondence: Ix8198@sohu.com

'Zhi-ping Zhang and Jun-tao Zhang contributed equally to this work. College of Veterinary Medicine, Henan Agricultural University, Zhengzhou 450046, China
}

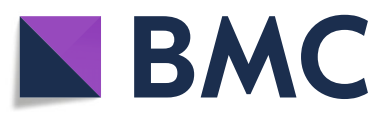

( The Author(s). 2020 Open Access This article is licensed under a Creative Commons Attribution 4.0 International License, which permits use, sharing, adaptation, distribution and reproduction in any medium or format, as long as you give appropriate credit to the original author(s) and the source, provide a link to the Creative Commons licence, and indicate if changes were made. The images or other third party material in this article are included in the article's Creative Commons licence, unless indicated otherwise in a credit line to the material. If material is not included in the article's Creative Commons licence and your intended use is not permitted by statutory regulation or exceeds the permitted use, you will need to obtain permission directly from the copyright holder. To view a copy of this licence, visit http://creativecommons.org/licenses/by/4.0/. The Creative Commons Public Domain Dedication waiver (http://creativecommons.org/publicdomain/zero/1.0/) applies to the data made available in this article, unless otherwise stated in a credit line to the data.

possess self-renewal and the capacity to differentiate into a wide variety of cell types, including ectoderm, mesoderm, and endoderm. Since ESCs were first successfully isolated [1-3], scientists have gradually focused on ESC research fields, including regenerative medicine, drug selection, and animal conservation. However, the destruction of embryos raises ethical issues [4]. Furthermore, immune responses of human ESCs have to be faced in clinical use, despite possessing immune-privileged properties [5]. Therefore, innovating alternative ways to 
obtain ESCs has become highly sought for personalized medicine.

In 1958, a cloned frog was obtained by using the injection of somatic cell nuclei into Xenopus oocyte [6], demonstrating that batrachian oocytes were capable of reprogramming somatic cells. When sheep and mice cloned by SCNT were successfully bred, mammalian oocytes were also shown to be able to reprogram somatic donor nuclei to a pluripotent state [7-9]. These great advances evoke the desire for the application of the SCNT technique in animal breeding and even in endangered animal conservation [10]. Reprogramming somatic cells into ESCs by oocytes has also been envisioned as an approach for generating patient-matched SCNTESCs for specific therapies and circumventing immune rejection by the host $[11,12]$. The genetically totipotent features of SCNT-ESC lines have been verified [13-17].

However, animal cloning is inefficient due to faulty epigenetic reprogramming, which in turn dysregulates gene expression [17-22]. A total of $\sim 9 \%$ of the dysregulated genes in SCNT-derived placenta were associated with transcriptomic reprogramming errors [23], which caused cloned animals to have shorter lifespans, most likely due to respiratory failure, hepatic failure, abnormal kidney development, liver steatosis, and large offspring syndrome [20, 24, 25]. All of the developmental abnormalities suggest that reprogramming of donor nuclei may not be fully completed by SCNT [26, 27], disturbing the gene expression patterns [28]. The reconstruction complexity and oocyte dependency of SCNT prompt the exploration of alternative approaches for somatic cell reprogramming. In addition to oocytes, pluripotent cells can dedifferentiate somatic cells by fusion and activate genes (such as the Oct4 gene) that are not expressed in adult cells. Therefore, ESCs or oocytes also contain factors that can confer totipotency or pluripotency to somatic cells [29-32]. Transcription factors, such as Oct3/4 [33, 34], Sox2 [35], and Nanog [36, 37], were confirmed to be effective in the maintenance of pluripotency in both early embryos and ESCs. Some genes, such as Stat3 [38, 39], E-Ras [40], c-Myc [41], Klf4 [42], and $\beta$-catenin [43], contributed to the long-term maintenance of the ES cell phenotype and rapid proliferation in vitro. A landmark advance reported that mouse pluripotent stem cells (iPSCs) were directly generated from fibroblast cultures by retroviral transduction of four transcription factors, Oct3/4, Sox2, Klf4, and c-Myc (named the Yamanaka factors) [44]. Subsequently, iPSCs were derived in several species, including humans [45-47] and rhesus monkeys [48], and the iPSCs have normal karyotypes and telomerase activity, express ES cell surface markers and genes, and maintain the developmental potential to differentiate into the three primary germ layers [49]. Similarly, iPSCs were derived from nearly all somatic cell populations, such as keratinocytes [50], neural cells [51, 52], stomach and liver cells [53], melanocytes [54], and lymphocytes [55], via various vectors [56]. To eliminate the risk of genomic integration and insertional mutagenesis, recent methodological improvements, such as treatment with microRNAs [57], synthetic mRNA modified [56], and valproic acid [58] as well as stimulus-triggered acquisition of pluripotency (transient low-pH stressor) [59] and chemically smallmolecule compounds [60], enhance the efficiency of reprogramming, reducing genomic modifications. These concentrated gains demonstrate an increasing number of reprogramming strategies, but these achievements also hint that the transcription network governing pluripotency is unclear. Less than $3 \%$ of somatic cells give rise to iPSC colonies. iPSCs are heterogeneous and highly diverse compared to ESCs due to epigenetic memory [61, 62] and epigenetic dynamics [63], which exhibit features of incomplete reprogramming and present limitations in disease modeling and personalized medicine [64]. Most iPSCs exhibit particular defects, such as poor quality of differentiation, low growth rate, aberrant transcription, disrupted DNA methylation and chromatin regulation, or chimeric animal contribution $[62,65,66]$. Theoretically, iPSCs can effectively overcome autologous immune rejection, but in contrast to derivatives of ESCs, abnormal gene expression in some cells differentiated from iPSCs can induce a $\mathrm{T}$ cell-dependent immune response in syngeneic recipients [67]. iPSCs differed and retained residual DNA methylation patterns typical of parental somatic cells, and the differentiation and epigenetic state of the donor nucleus influence reprogramming efficiency [68]. iPSCs derived from different somatic cell sources show different capacities of reprogramming and differentiation; for example, progenitor cells are more efficient at giving rise to iPSCs than terminally differentiated cells $[55,69]$. iPSCs are prone to (epi) genetic instability during in vitro culture [70]. In contrast, the differentiation and methylation characteristics of SCNT cells were more similar to those of classical ESCs than iPSCs [61]. Although iPSC reprogramming is technically simpler, it is less efficient and slower than SCNT and cell fusion [71]. There are strict standards regarding the specificity, efficiency, kinetics, and safety of stem cells for clinical use, so reprogramming, methodological improvements, or fundamental changes in SCNT and iPSCs are considered.

\section{Definition of double sperm cloning}

Compared with iPSCs, SCNT-ESCs bear "fewer abnormalities" and exhibit characteristics that "more closely resemble genuine embryonic stem cell" traits, which may favor their use as therapies in treating particular conditions [72]. Therefore, many challenges must be 
faced for establishing the standardized procedure of reprogramming somatic cells by SCNT or iPSCs, which may be an elusive topic. Therefore, in addition to paying more attention to improving the reprogramming efficiency in somatic cells by SCNT and iPSCs, we should innovate other reprogramming alternatives that are relatively easy to standardize to fulfill concerns about specificity, efficiency, and safety in clinical use. Here, we define double sperm cloning (DSC), which is based on androgenetic diploid embryos. Strictly defined, DSC involves two capacitated sperm, which are sorted by sex via flow cytometry, that are then injected into denucleated oocytes by intracytoplasmic sperm injection (ICSI). Afterward, the fertilized embryos are cultured to form blastocysts and to derive ESCs. This strategy offers a promising method for regenerative medicine and animal breeding, and it possesses unique superiority to SCNT and iPSC methods. This article mainly illuminates the realization, performance, advantages, and application of DSC, and it highlights the opportunities and challenges compared to SCNT and iPSCs.

\section{Operability of ESCs derived from double sperm cloning embryos}

To avoid stability and efficiency issues with somatic cells reprogrammed by SCNT and iPSCs, we used a distinct way to harvest ESCs from the embryo by the DSC method. DSC involves several key steps, including ICSI and the use of very mature enucleated oocytes. For example, metaphase II oocyte cytoplasts are easily enucleated with mechanical [73] or chemically assisted methods [74, 75]. With DSC, the constructed embryos develop into blastocysts and ESCs can be isolated from their inner mass cells (Fig. 1). The strategy not only simulates natural fertilization but also makes good use of established techniques, such as ICSI and oocyte enucleating. It guarantees a normal diploid karyotype of the reconstructed embryo.

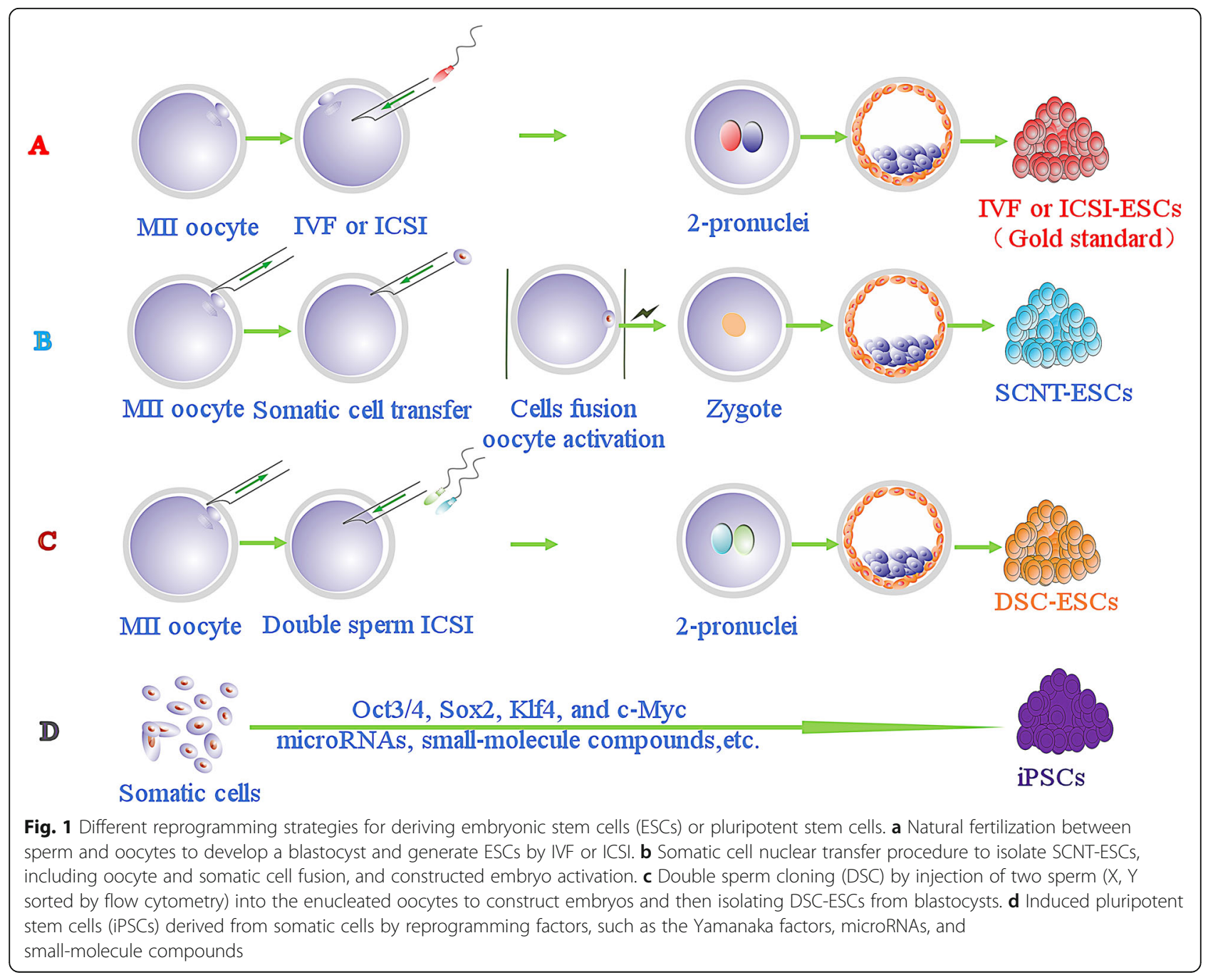


The oocyte is the best system for supporting the reprogramming of a homogeneous cell. In fact, natural fertilization is also reprogramming of oocytes to sperm, and reprogramming of somatic nuclei is not as efficient as that of sperm nuclei. Sperm chromatin is epigenetically modified to be adequate for early embryonic development, while somatic nuclei do not have such modifications. Moreover, epigenetic memories encoded in sperm chromatin are transgenerationally inherited, implying unique roles of sperm [76]. In human clinical reproduction, an inspection of pronucleus formation is routine for selecting zygotes with exact two pronuclei after IVF; if zygotes with more than two pronuclei are observed, the embryo must be discarded due to polyspermic fertilization. Therefore, all of these results illustrate that oocytes can simultaneously reprogram two sperm through DSCs. Other procedures refer to sophisticated programs for deriving ESCs from embryos [3, 11]. Hence, the DSC strategy could be used to produce superior ESCs in theory and practice.

\section{Oocyte activation by sperm factors}

In addition to oocyte quality being a prominent part of reprogramming, several steps in SCNT operation, including spindle removal, donor cell fusion, and cytoplast activation, are also critical for cellular reprogramming to blastocyst development [11]. However, natural fertilization involves fewer steps than SCNT does, as the entry of only one sperm triggers oocyte activation, which is critical for the completion of meiosis and the initiation of mitotic divisions. In both sperm and oocytes, activation is also critical for the oocyte cytoplasm to acquire reprogramming and metabolic activity, which is necessary to support subsequent embryo development [77].

Sperm factors are thought to initiate oocyte activation through oscillations in $\mathrm{Ca}^{2+}$ at fertilization by spermoocyte fusion [78-81], and the demethylation process is facilitated either by a sperm-derived factor or by male pronuclear chromatin composition [82], and calcium plays a pivotal role in this process. The injection of sperm cytosolic extracts into oocytes triggers a prolonged series of $\mathrm{Ca}^{2+}$ oscillations similar to those seen at fertilization [83]. In mouse and human ICSI, a prolonged series of $\mathrm{Ca}^{2+}$ oscillations is also accompanied by sperm factors. Therefore, oocytes in DSCs are also activated by sperm factors similar to ICSI and IVF. However, somatic cells lack these factors, and oocytes must be activated in other ways, such as chemical activation or electric activation in SCNT. Reactive oxygen species levels and $\mathrm{Ca}^{2+}$ oscillations differed between sperm-activated oocytes and parthenogenetically activated oocytes [83, 84]. Therefore, oocyte activation in DSC is more natural and superior for embryo development than what is observed in SCNT. The DSC procedure is simple because it does not require donor cell artificial fusion. Additionally, preprocessing of sperm and somatic cells is different between SCNT and DSC.

\section{Sperm superiority of reprogramming}

Sperm are highly differentiated, transcriptionally inert cells with minimal cytoplasm, and they contain a suite of unique RNAs that are delivered to oocytes. These RNAs are likely involved in many different processes, including genome recognition, early embryonic development, and epigenetic transgenerational inherence [85]. One of the biggest differences between sperm and somatic cells is the fact that somatic cell DNA is wrapped around histones, whereas sperm DNA is tightly packed by protamines, which condenses sperm DNA to one sixth the size of the mitotic chromosomes and carefully protects their DNA [86]. At fertilization, the highly condensed and transcriptionally inert chromatin of the sperm is remodeled into the decondensed and transcriptionally competent chromatin of the male pronucleus [87]. Sperm also carry numerous paternal mRNAs to oocytes at fertilization, facilitating early development [88-90]. Sperm is important for the first cell division and can influence the pattern of embryonic gene expression and even phenotypes of the progeny [91]. Epigenetic marks in sperm are extensive and are correlated with developmental regulators [92]. All of the sperm chromatin features are likely to support embryonic development after fertilization. Somatic chromatin does not have such "fine-tuning" for correct embryonic gene expression. Therefore, embryos generated from SCNT often show abnormal reprogramming events compared to fertilized embryos [76], and the cell cycle state of the donor as well as their level of differentiation may be important determinants of reprogramming efficiency. Scientists compared the differences between iPSCs and ESCs and found persistent donor cell gene expression and epigenetic memories in iPSCs [66, 93-96]; however, sperm express fewer genes and carry fewer epigenetic marks than iPSCs. Therefore, the superiority of DSC is the result of sperm chromatin features, which may be useful in embryo development.

\section{Mitochondrial features}

Mitochondrial DNA (mtDNA) possesses unique properties, including high copy numbers, maternal inheritance, lack of recombination, and high mutation rate. Many mtDNA mutations have been found to be related to aging, neurodegeneration, and tumorigenesis [97-99]. Aged somatic cells might show high susceptibility to nuclear and mitochondrial genome instability [100]. Hypothetically, in reprogrammed somatic cells from patients to generate pluripotent stem cells for therapeutic application, mtDNA mutations of the somatic cell must be 
evaluated, including analysis of a broad spectrum of degenerative diseases associated with mutations in mtDNA, which are unlikely to be amenable to iPSCbased therapies due to the persistence of the somatic cell mtDNA mutations [101]. Furthermore, mature oocytes contain more than 150,000 copies of mtDNA, which is at least an order of magnitude greater than the number in most somatic cells, and sperm contain only approximately 100 copies [102]. ICSI performed with mature sperm does not alter the uniparental pattern of inheritance of mtDNA, and mtDNA is selectively degraded through a selective silencing process that occurs early in development [103]. In mice, most of the offspring carried donor cell-derived mtDNA that constituted as much as $13.1 \%$ of the total [104]. Therefore, the small amount of mtDNA provided via sperm by DSC cannot disturb embryo development and suggests its safety in clinical use, such as in iPSCs and somatic cells reprogrammed by SCNT.

\section{Telomere importance}

Telomeres are protective end complexes at the end of mammalian chromosomes. Telomere length gradually shortens as stem cells divide to produce differentiated cells, eventually resulting in replicative senescence with aging. Sometimes, the loss of telomeres can lead to potentially maladaptive cellular changes, block cell division, and interfere with tissue replenishment, especially in diseases of human aging and in some aging-related processes [105]. Telomeres and telomerase are the main components of the stem cell "ignition" mechanism for tissue regeneration and provide a way to restrain cancer and delay aging [106]. Telomere lengths are highly correlated with the developmental pluripotency of ESCs. For example, ESCs with long telomeres exhibit authentic developmental pluripotency, as evidenced by the generation of complete ESC-derived offspring as well as germline-competent chimeras [107]. Cells with short telomeres cannot be reprogrammed to generate iPS cells despite their normal proliferation rates [108]. Therefore, telomere length is considered a valuable marker for evaluating stem cell pluripotency. Telomerase is strongly expressed in ESCs and is inactive in most somatic cells. Therefore, telomeres and telomerase must be key factors in somatic reprogramming to stem cells. Telomeric chromatin is remodeled, and telomeres are elongated by telomerase during nuclear reprogramming. For example, telomerase activity and telomere lengths were increased in SCNT-derived blastocysts compared to donor cells $[109,110]$. This suggests that oocytes possess a perfect reprogramming cytoplasm for donor cells. Similarly, compared to differentiated cells, iPSCs also have longer telomeres with increasing passages until telomeres reach a length that is characteristic of ES cells [111]. SCNT- mediated reprogramming mitigates telomere dysfunction and mitochondrial defects to a greater extent than iPSCbased reprogramming [112], and ESCs have greater differentiation potential and self-renewal capacity than iPSCs. Telomeres in mammalian male germ cells progressively increase in length from spermatogonia to sperm during spermatogenesis. However, telomerase activity is gradually downregulated during germ cell differentiation from spermatogonia to sperm, and no telomerase activity occurs in the spermatozoa [113]. Therefore, in DSC, two sperm with long telomeres are introduced into oocytes with stronger reprogramming capacity and higher telomerase activity, which is similar to the situation in IVF embryos. Theoretically, we can derive DSC-ESCs with normal telomeres from the DSCderived blastocyst.

\section{Storage superiority of sperm}

This method may be used to obtain embryos and derive specific DSC-ESCs. So, the sperm bank has a new function. Compared to cell cryopreservation for clinical use, sperm cryopreservation is simple and completely practicable, and its maintenance cost is much lower than that of somatic cells. Theoretically, DSC-ESCs could be used to cure donor disease, and they could also be used to treat his children. Personalized DSC-ESCs can offer two types, $\mathrm{XY}$ and $\mathrm{XX}$, for donor and his baby, whose chromosome only comes from their father (Fig. 2).

\section{Progress of DSC}

At present, scientists mainly focus on the establishment of haploid embryonic stem cells [114, 115]. In 2012, Hui Y [116] and Wei Li [117] reported the derivation of androgenetic haploid ES (ahES) cells from androgenetic blastocysts, and they obtained live mice upon injection of ahES cells into MII oocytes. In 2015, Ding C produced androgenetic haploid human embryos by injecting a single spermatozoon into an enucleated human oocyte and established human androgenetic embryonic stem (hAGES) cell lines from androgenetic embryos, which exhibit typical features of human ESCs [118]. The hAGES cells maintained a sperm methylation pattern to a certain extent. The ahES cells could produce viable and fertile progenies after intracytoplasmic injection into mature oocytes [118]. These achievements are similar to what can be achieved with DSC-ESCs. All of these studies show that enucleated oocytes are completely capable of reprogramming one sperm and gaining ahES cells exhibiting typical features of ESCs. In 1984, James McGrath constructed diploid mouse embryos with two male pronuclei by transplantation of pronuclei and found that they could not develop to term. They concluded that a diploid genome derived from only one of the two parental sexes is incapable of supporting 


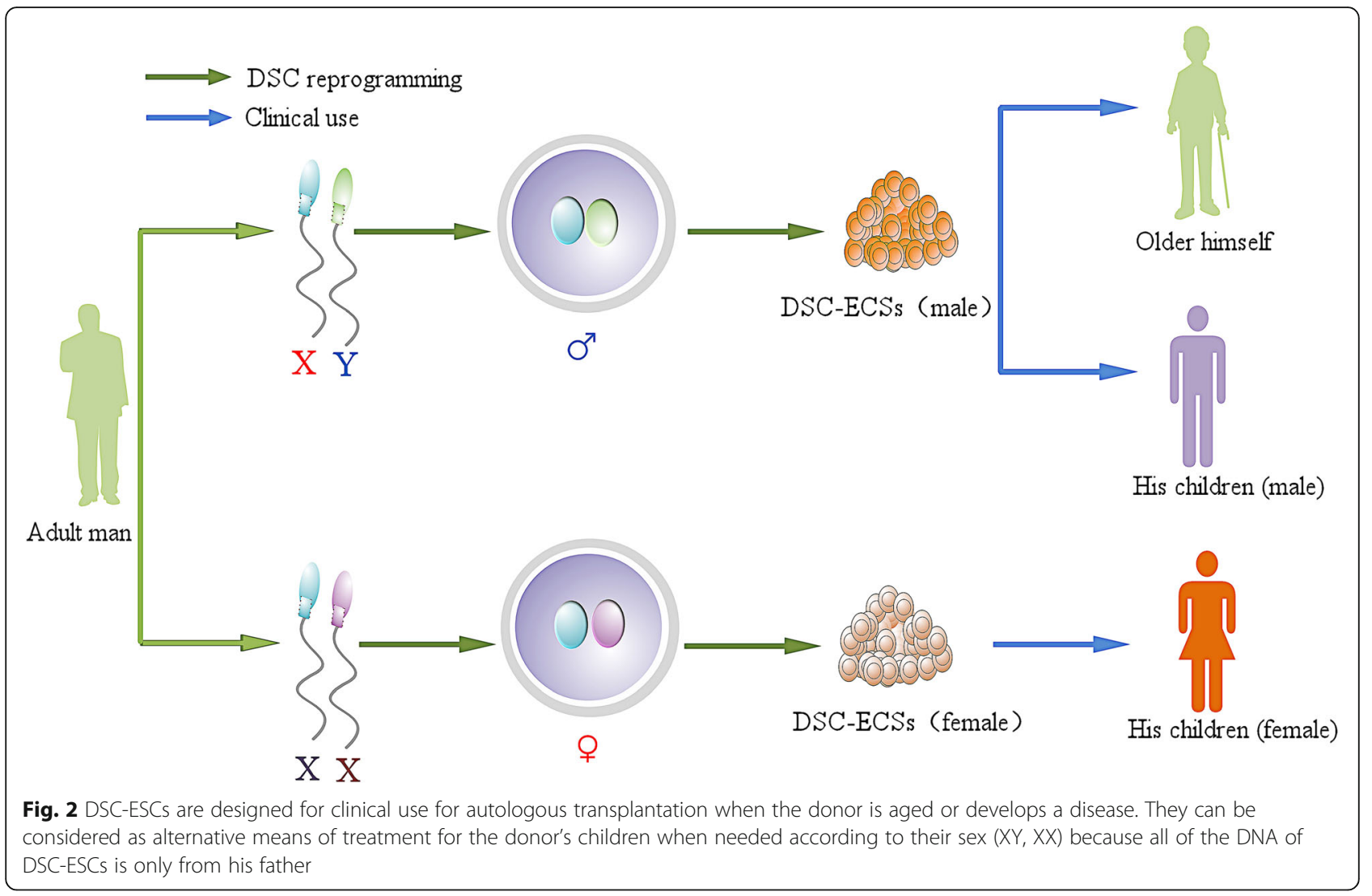

complete embryogenesis [118], and imprinted genes showed paternal bias in the placenta but not in the fetus [119]. Kono et al. increased the concentration of sperm use in fertilization with enucleated oocytes, resulting in $35-45 \%$ efficiency of fertilizing eggs, which produced heterozygous bispermic androgenones, and $43 \%$ of embryos developed to blastocysts [120]. Lagutina et al. reported that $31 \%$ of bovine diploid androgenetic embryos (DSC embryos) could develop into blastocysts, which was a rate similar to that of IVF control embryos (35\%), and following the transfer of diploid androgenetic embryos, a pregnancy could be established and maintained up to day 28 [121]. In sheep, Matsukawa et al. reported the use of IVF in producing diploid androgenetic embryos resulted in no significant difference in early cleavage and morula, but the blastocyst formation rate was significantly lower. However, diploid androgenetic embryos produced by pronuclear exchange developed to the blastocyst stage at a higher proportion (19\%) [122]. Theoretically, two Y chromosome embryos cannot regularly develop due to the lack of the $\mathrm{X}$ chromosome; if the "aberrant" androgenetic embryo (YY) can be ruled out, the blastocyst formation rate is higher. Furthermore, the developmental potential of haploid embryos is significantly impaired relative to diploid embryos; Latham et al. discovered subtle alterations in the gene expression in haploid androgenones, such as the lack of repression of the Pgk1 gene, from what is seen in diploid androgenones, which experience $\mathrm{X}$ chromosome inactivation [123]. The blastocyst formation rate from androgenetic diploid embryos is higher than that from androgenetic haploid embryos ( $43 \%$ vs $11 \%$, respectively, in mice [120] and $31 \%$ vs $1.8 \%$, respectively, in bovines [121]). Therefore, we should derive more DSC blastocysts (androgenetic diploid embryos) for isolating ESCs. In 2009, Teramura et al. established authentic ESCs from androgenetic diploid mouse embryos by IVF from two sperm and a denucleated oocyte by taking advantage of adjusting the sperm concentration and the zona pellucida incision [124], and they induced differentiation of mouse AgESCs and observed derivation of neural cells, cardiomyocytes, and hepatocytes in vitro and found that an embryoid body generated from the cells could engraft in theoretically MHC-matched strains [124]. Dinger et al. also observed that AgESC-derived neural progenitor/stem cells do not differ from normal neural progenitor/stem cells in their self-renewal and neural differentiation potential in mice in vivo and in vitro and exhibited fidelity regarding the expression of six imprinted genes analyzed, though the expression of Ube3a had changed [125]. Therefore, DSC-ESCs is promising. 


\section{Clinical therapy and ethics related to DSC-ESCs}

Regardless of the stem cell type, before the cells can be used in regenerative medicine, the safety and standard procedures must be established. For natural embryos, the procedure of establishing ESCs is easy to standardize. Once efficient differentiation protocols for the generation of a target cell lineage are established, ESCs are expected to stably supply large amounts of cell substrates for use in cell-based therapies, which have greater differentiation and replication ability than somatic stem cells. Human ES cell-based products were recently evaluated in clinical trials in the USA. However, human ESCs are generated by the destruction of human embryos, so there are ethical issues [126]. ESCs from IVF embryos are allogeneic to the patients that would receive them for treatment. SCNT-ESCs and iPSCs can be derived from the donors' cell of a patient; they are autologous cells with less immunogenicity, but they are prone to epigenetic and transcriptional aberrations. These cells acquire genetic modifications in addition to epigenetic modifications, and extensive genetic screening should become a standard procedure to ensure safety for clinical use [127]. The protocols to produce ESCs involve many steps or different methods, as mentioned above, making this process difficult to standardize. Compared to somatic cells reprogrammed by SCNT and iPSCs, the DSC approach produces ESCs with a set of standard procedures, and DSC-ESCs are matched with the patients (Fig. 2). Herein, by clarifying the advantages and disadvantages of the current reprogramming systems, we may be providing an effective strategy for generating clinical-grade cells.

\section{Animal breeding by DSC}

Currently, prevalent livestock animal breeding mainly follows the traditional pattern of progeny testing, which requires many years of breeding unique traits and gains stabilized genetic characteristics following a strict breeding program. With the help of reproductive technology, including artificial insemination and embryo transfer, improved breeds could be popularized for commercial applications. The emergence of animal cloning provides a promising method for breed conservation. In cloned cattle, blood profiles and other indicators of general physiological function, such as growth rate, reproduction, rearing of offspring, and milk production, are all within normal phenotypic ranges [128]. If the challenges of DSC are successfully overcome with increased improvements to the methods, this would provide a great tool for use in animal breeding. Not only can we make use of two sperm (X and $\mathrm{Y}$ ) from one male individual, similar to SCNT, but we can also apply two $\mathrm{X}$ sperm from one male in the production of female offspring. Furthermore, we can establish a new animal breeding system by assembling the sperm of different sexes from diverse breeds (Fig. 3).

\section{Challenges of DSC, SCNT, and iPSCs}

Genomic imprinting occurs via an epigenetic mechanism, which means that some imprinted genes are only expressed from their maternal allele, while others are only expressed from their paternal allele. Approximately $1 \%$ of human genes are normally expressed from only the maternally or paternally inherited gene copy [129]. Mammals are biparental diploid organisms, and both maternal and paternal genomes are required for normal development. Genomic imprinting affects several dozen mammalian genes and directs the expression of those genes by DNA methylation, resulting in the expression of either the maternal or paternal allele [130]. DNA methylation memory establishment, maintenance, and erasure are carefully balanced by molecular machinery that is highly conserved among vertebrates [131]. Most imprinted genes contain differentially methylated regions (DMRs) between the maternal and paternal alleles [132]. Disrupted imprinting is associated with significant pathologies [133], such as disruption of the transfer of nutrients [134]. Methylation patterns are reprogrammed genome-wide in embryos and generate cells with broad developmental potential. Epigenetic reprogramming is critical for the imprinting process [135]. The low success rate of SCNT in cloning is largely due to imprinting problems. The percentage of the DMR that was methylated in imprinted genes (XIST and H19) was significantly decreased, and short-lived cloned bovines exhibited more severe aberrant methylation changes in the examined imprinted genes [136]. In certain SCNTESC lines, DNA methylation patterns of a paternally imprinted gene, H19, displayed distinct abnormalities and appeared to be very dynamic; maternally imprinted genes, Mest and Peg3, showed relatively stable methylation patterns in ES cells [137]. The altered expression of imprinted genes associated with SCNT is also caused by changes in histone modifications [138]. Similarly, imprinting errors are observed in iPSCs, suggesting that these epigenetic anomalies are related to the reprogramming process and could be directly responsible for the variable phenotypes and low success rates of both cloning and iPS derivation procedures [139]. Certainly, the epigenetic abnormalities detected in iPSCs are not specific to transcription factor-mediated reprogramming [140]. It is difficult to program via SCNT and iPSC induction, and it is also a challenge to cause two sperm from paternal genomes to construct a DSC embryo with the correct reprogramming procedure. Epigenetic studies have demonstrated changes to the DSC procedure that are necessary for mammalian physiology [141]. In DSC embryos, two sperm contain some similar 


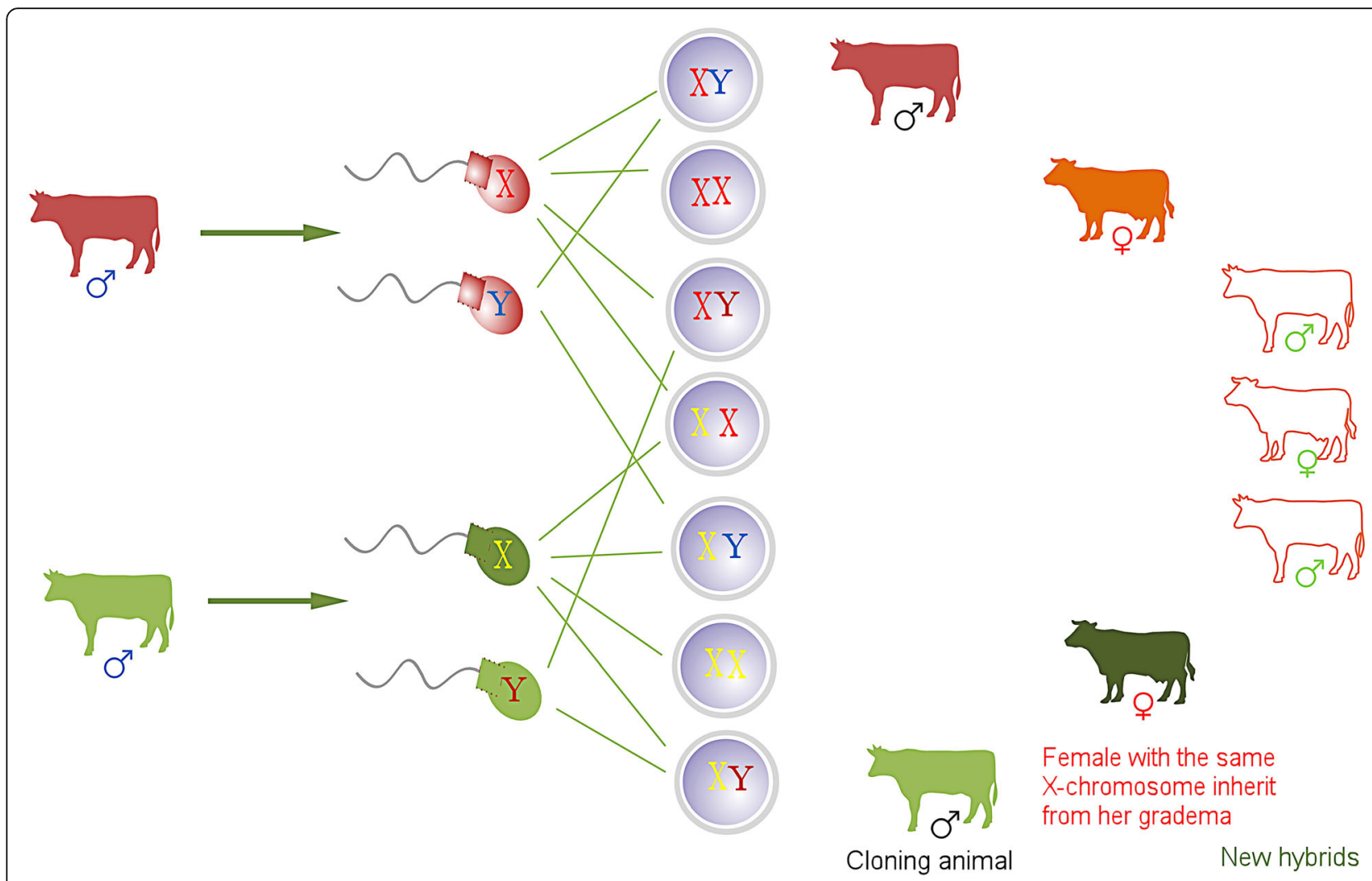

Fig. 3 Animal breeding with DSC: cloned animals are bred by DSC (his one XY); female animals inherit the X chromosome from the female animal that parented her parent; breeding new hybrids by DSC

imprinted genes disturbing embryo development. During oocyte reprogramming, imprinted genes could be coordinated to function in accordance with evolutionary conservation [142]. For example, the impact of external environments also results in DNA methylation alterations. Mikhael reported that H19/IGF2 imprinting may be epigenetically stable after reprogramming in cloned horses [143]. Fortunately, DSC embryos can develop blastocysts at a high rate and even establish early pregnancy [120, 121]. Mouse androgenetic diploid ESCs have been established and can differentiate into other cell types [124]. We believe that all of these studies help establish DSC-ESCs as a promising tool for regenerative medicine.

In mammals, epigenetic marks on the $\mathrm{X}$ chromosomes are involved in dosage compensation, and transcriptional silencing of one of the two X chromosomes randomly occurs in female cells during late blastocyst development [144]. Incomplete nuclear reprogramming in cloning animals may affect both random and imprinted XCI [145], and many genes on the X chromosome are specifically downregulated [104]. In iPSC reprogramming, mouse iPSCs exhibit X chromosome activation (XCA) of two chromosomes, while there is XCA of only one in humans [146]. Thus, there are different X chromosome statuses in reprogramming. XCI has been shown to vary widely in human female iPSCs and ESCs [147]. Therefore, if we derive human ESCs by DSC, dosage compensation must be accounted for; specifically, the XCI status must be considered in female cells, and in male (XY) DSC-ESCs, $\mathrm{X}$ chromosome reactivation must be addressed. The DSC method offers a sex chromosome combination (XX, XY, and $\mathrm{YY}$ ) for exploring the dosage compensation mechanism.

\section{Conclusion}

Stem cells have the capacity for self-renewal and differentiation into various cell lineages in regenerative medicine. ESCs are the classic representative of stem cells. To meet conditions related to efficient reprogramming, sufficient cell yield, ethical concerns, specificity, and safety, SCNT-ESCs and iPSCs have been generated to produce pluripotent cells with the hope of clinical use. For example, oocytes can reprogram a somatic cell in less than 3 days while retaining an intact genome and epigenome [148], and SCNT-ESCs showed lower chromosome mutation [149]. In addition, accumulating preclinical data also support the safety and efficacy of iPSCs [150]. These emerging technologies motivate new research trends with many exciting achievements, but low reprogramming efficiency and genomic modification steps still 
restrict the clinical use of these cells [151]. For example, iPSCs always possess somatic-coding mutations [127]. SCNT and iPSCs are not perfect, exhibiting genetic and epigenetic abnormalities and immunogenicity [150]. In addition, their standardization of reprogramming is difficult. Donor cells come from variable differentiated cells, and reprogramming procedure also varies. Donor cells from patients can have mtDNA mutations or exhibit signs of aging. Therefore, we propose a promising alternative, double sperm cloning, to produce ESCs that avoid the abovementioned disadvantages. Theoretically, DSC has many advantages, such as enabling sperm storage, standardization, and specificity as well as maintaining mtDNA quality being a simple procedure and reprogramming process that is similar to natural fertilization. The successful mouse DSC-ESCs strongly support this strategy. However, no live animal was born from DSC, perhaps due to epigenetic modifications. These issues also exist in somatic cells reprogrammed by SCNT or iPSCs. Scientists have ignored such issues; until Dolly the sheep was born, most scientists considered oocyte reprogramming of somatic cells to an embryonic state an impossibility. Most studies have focused on genetics to illuminate why this was impossible. Recently, more studies have been performed on uniparental embryos and have derived uniparental haploid ESCs. DSC deserves more attention and should be studied to achieve goals in medicine and animal breeding.

\section{Abbreviations}

ESCs: Embryonic stem cells; SCNT: Somatic cell nuclear transfer; IPSCs: Induced pluripotent stem cells; DSC: Double sperm cloning; ICSI: Intracytoplasmic sperm injection; LIF: Leukemia inhibitory factor; bFGF: Basic fibroblast growth factor; PLC ; Phospholipase C, zeta; PAWP: Post-acrosomal sheath WW domain-binding protein; mtDNA: Mitochondrial DNA; hAGES: Human androgenetic embryonic stem; DMRs: Differentially methylated regions; $X \mathrm{Cl}$ : X chromosome inactivation; XCA: X chromosome activation

\section{Acknowledgements}

The authors sincerely acknowledge and thank the American Journal Experts for the editing service for improving the English language, grammar, and spelling of our manuscript.

\section{Authors' contributions}

ZPZ and JTZ wrote the manuscript. SCH, XYH, and LXD edited the manuscript. All authors read and approved the final manuscript.

\section{Funding}

This review was supported by the Program of the National Beef Cattle and Yak Industrial Technology System (CARS-37), National Natural Science Foundation of China (No.31001096), Natural Science Foundation of Henan Province (162300410145), and Project of Education Department of Henan Province (16A230022).

\section{Availability of data and materials}

Not applicable.

Ethics approval and consent to participate Not applicable.
Consent for publication

Not applicable.

\section{Competing interests}

The authors declare that they have no competing interests.

Received: 13 June 2020 Revised: 12 August 2020

Accepted: 27 August 2020 Published online: 07 September 2020

\section{References}

1. Martin GR. Isolation of a pluripotent cell line from early mouse embryos cultured in medium conditioned by teratocarcinoma stem cells. Proc Natl Acad Sci U S A. 1981;78:7634-8.

2. Evans MJ, Kaufman MH. Establishment in culture of pluripotential cells from mouse embryos. Nature. 1981;292:154-6.

3. Thomson JA, Itskovitz-Eldor J, Shapiro SS, et al. Embryonic stem cell lines derived from human blastocysts. Science. 1998;282:1145-7.

4. Hayakawa T, Aoi T, Umezawa A, et al. A study on ensuring the quality and safety of pharmaceuticals and medical devices derived from the processing of autologous human somatic stem cells. Regen Ther. 2015;2:57-69.

5. Li L, Baroja ML, Majumdar A, et al. Human embryonic stem cells possess immune-privileged properties. Stem Cells. 2004;22:448-56.

6. Gurdon JB, Elsdale TR, Fischberg M. Sexually mature individuals of Xenopus laevis from the transplantation of single somatic nuclei; 1958.

7. Wilmut I, Schnieke AE, MCWhir J, Kind AJ, Campbell KHS. Viable offspring derived from fetal and adult mammalian cells. Nature. 1997;385:810-3.

8. Wakayama T, Perry AC, Zuccotti M, Johnson KR, Yanagimachi R. Full-term development of mice from enucleated oocytes injected with cumulus cell nuclei. Nature. 1998:394:369-74.

9. Rideout WM, Eggan K, Jaenisch R. Nuclear cloning and epigenetic reprogramming of the genome. Science. 2001;293:1093-8.

10. Keefer CL. Artificial cloning of domestic animals. Proc Natl Acad Sci. 2015; 112:8874-8

11. Tachibana M, Amato $P$, Sparman M, et al. Human embryonic stem cells derived by somatic cell nuclear transfer. Cell. 2013;153:1228-38.

12. Yang $X$, Smith $S L$, Tian $X C$, et al. Nuclear reprogramming of cloned embryos and its implications for therapeutic cloning. Nat Genet. 2007;39:295-302.

13. Wakayama $T$, Tabar $V$, Rodriguez I, et al. Differentiation of embryonic stem cell lines generated from adult somatic cells by nuclear transfer. Science. 2001:292:740-3.

14. Wakayama S, Ohta H, Kishigami S, et al. Establishment of male and female nuclear transfer embryonic stem cell lines from different mouse strains and tissues. Biol Reprod. 2005;72:932-6.

15. Kawase E, Yamazaki Y, Yagi T, Yanagimachi R, Pedersen RA. Mouse embryonic stem (ES) cell lines established from neuronal cell-derived cloned blastocysts. Genesis. 2000;28:156-63.

16. Munsie MJ, Michalska AE, O'Brien CM, et al. Isolation of pluripotent embryonic stem cells from reprogrammed adult mouse somatic cell nuclei. Curr Biol. 2000;10:989-92.

17. Hochedlinger $K$, Jaenisch R. Monoclonal mice generated by nuclear transfer from mature B and T donor cells. Nature. 2002:415:1035-8.

18. Wakayama T, Yanagimachi R. Cloning of male mice from adult tail-tip cells. Nat Genet. 1999:22:127-8.

19. Humpherys $D$, Eggan $\mathrm{K}$, Akutsu $\mathrm{H}$, et al. Abnormal gene expression in cloned mice derived from embryonic stem cell and cumulus cell nuclei. Proc Natl Acad Sci. 2002;99:12889-94.

20. Ogonuki N, Inoue K, Yamamoto $Y$, et al. Early death of mice cloned from somatic cells. Nat Genet. 2002;30:253-4.

21. Tamashiro KL, Wakayama T, Akutsu H, et al. Cloned mice have an obese phenotype not transmitted to their offspring. Nat Med. 2002;8:262-7.

22. Gurdon J, Byrne J, Simonsson S. Nuclear reprogramming and stem cell creation. Proc Natl Acad Sci. 2003;100:11819-22.

23. Salilew-Wondim D, Tesfaye D, Hossain M, et al. Aberrant placenta gene expression pattern in bovine pregnancies established after transfer of cloned or in vitro produced embryos. Physiol Genomics. 2013:45:28-46.

24. Hill JR. Incidence of abnormal offspring from cloning and other assisted reproductive technologies. Annu Rev Anim Biosci. 2014:2:307-21.

25. Rutigliano HM, Wilhelm A, Hall J, et al. Cytokine gene expression at the maternal-fetal interface after somatic cell nuclear transfer pregnancies in small ruminants. Reprod Fertil Dev. 2017;29(4):646-57. 
26. Beyhan Z, Forsberg E, Eilertsen K, Kent-First M, First N. Gene expression in bovine nuclear transfer embryos in relation to donor cell efficiency in producing live offspring. Mol Reprod Dev. 2007;74:18-27.

27. Inoue K, Ogonuki N, Kamimura S, et al. Loss of H3K27me3 imprinting in the Sfmbt2 miRNA cluster causes enlargement of cloned mouse placentas. Nat Commun. 2020;11:2150.

28. Dindot SV, Farin PW, Farin CE, et al. Epigenetic and genomic imprinting analysis in nuclear transfer derived Bos gaurus/Bos taurus hybrid fetuses. Biol Reprod. 2004;71:470-8

29. Tada M, Tada T, Lefebvre L, Barton SC, Surani MA. Embryonic germ cells induce epigenetic reprogramming of somatic nucleus in hybrid cells. EMBO J. 1997;16:6510-20.

30. Tada M, Takahama Y, Abe K, Nakatsuji N, Tada T. Nuclear reprogramming of somatic cells by in vitro hybridization with ES cells. Curr Biol. 2001;11:15538.

31. Ying Q-L, Nichols J, Evans EP, Smith AG. Changing potency by spontaneous fusion. Nature. 2002:416:545-8.

32. Terada N, Hamazaki T, Oka M, et al. Bone marrow cells adopt the phenotype of other cells by spontaneous cell fusion. Nature. 2002;416:5425

33. Nichols J, Zevnik B, Anastassiadis K, et al. Formation of pluripotent stem cells in the mammalian embryo depends on the POU transcription factor Oct4. Cell. 1998;95:379-91.

34. Niwa H, Miyazaki J-I, Smith AG. Quantitative expression of Oct-3/4 defines differentiation, dedifferentiation or self-renewal of ES cells. Nat Genet. 2000; 24:372-6.

35. Avilion AA, Nicolis SK, Pevny LH, et al. Multipotent cell lineages in early mouse development depend on SOX2 function. Genes Dev. 2003;17:12640

36. Chambers I, Colby D, Robertson M, et al. Functional expression cloning of Nanog, a pluripotency sustaining factor in embryonic stem cells. Cell. 2003 113:643-55

37. Mitsui $K$, Tokuzawa $Y$, Itoh $H$, et al. The homeoprotein Nanog is required for maintenance of pluripotency in mouse epiblast and ES cells. Cell. 2003;113: 631-42

38. Matsuda T, Nakamura T, Nakao K, et al. STAT3 activation is sufficient to maintain an undifferentiated state of mouse embryonic stem cells. EMBO J. 1999:18:4261-9.

39. Niwa H, Burdon T, Chambers I, Smith A. Self-renewal of pluripotent embryonic stem cells is mediated via activation of STAT3. Genes Dev. 1998; 12:2048-60.

40. Takahashi K, Mitsui K, Yamanaka S. Role of ERas in promoting tumour-like properties in mouse embryonic stem cells. Nature. 2003;423:541-5.

41. Cartwright P, McLean C, Sheppard A, et al. LIF/STAT3 controls ES cell selfrenewal and pluripotency by a Myc-dependent mechanism. Development. 2005;132:885-96

42. Li Y, McClintick J, Zhong L, et al. Murine embryonic stem cell differentiation is promoted by SOCS-3 and inhibited by the zinc finger transcription factor Klf4. Blood. 2005:105:635-7.

43. Kielman MF, Rindapää M, Gaspar C, et al. Apc modulates embryonic stemcell differentiation by controlling the dosage of $\beta$-catenin signaling. Nat Genet. 2002;32:594-605.

44. Takahashi K, Yamanaka S. Induction of pluripotent stem cells from mouse embryonic and adult fibroblast cultures by defined factors. Cell. 2006;126: 663-76.

45. Takahashi K, Tanabe K, Ohnuki M, et al. Induction of pluripotent stem cells from adult human fibroblasts by defined factors. Cell. 2007;131:861-72.

46. Yu J, Vodyanik MA, Smuga-Otto K, et al. Induced pluripotent stem cell lines derived from human somatic cells. Science. 2007;318:1917-20.

47. Park I-H, Zhao R, West JA, et al. Reprogramming of human somatic cells to pluripotency with defined factors. Nature. 2008;451:141-6.

48. Liu H, Zhu F, Yong J, et al. Generation of induced pluripotent stem cells from adult rhesus monkey fibroblasts. Cell Stem Cell. 2008;3:587-90.

49. Stadtfeld M, Hochedlinger K. Induced pluripotency: history, mechanisms, and applications. Genes Dev. 2010;24:2239-63.

50. Aasen T, Raya A, Barrero MJ, et al. Efficient and rapid generation of induced pluripotent stem cells from human keratinocytes. Nat Biotechnol. 2008;26: 1276-84.

51. Eminli S, Utikal J, Arnold K, Jaenisch R, Hochedlinger K. Reprogramming of neural progenitor cells into induced pluripotent stem cells in the absence of exogenous Sox2 expression. Stem Cells. 2008;26:2467-74
52. Kim JB, Zaehres $H, W u$ G, et al. Pluripotent stem cells induced from adult neural stem cells by reprogramming with two factors. Nature. 2008;454:64650.

53. Aoi T, Yae K, Nakagawa M, et al. Generation of pluripotent stem cells from adult mouse liver and stomach cells. Science. 2008;321:699-702.

54. Utikal J, Maherali N, Kulalert W, Hochedlinger K. Sox2 is dispensable for the reprogramming of melanocytes and melanoma cells into induced pluripotent stem cells. J Cell Sci. 2009;122:3502-10.

55. Eminli S, Foudi A, Stadtfeld M, et al. Differentiation stage determines potential of hematopoietic cells for reprogramming into induced pluripotent stem cells. Nat Genet. 2009;41:968-76.

56. Warren L, Manos PD, Ahfeldt T, et al. Highly efficient reprogramming to pluripotency and directed differentiation of human cells with synthetic modified mRNA. Cell Stem Cell. 2010;7:618-30.

57. Li Z, Dang J, Chang K-Y, Rana TM. MicroRNA-mediated regulation of extracellular matrix formation modulates somatic cell reprogramming. RNA. 2014:20:1900-15.

58. Huangfu D, Maehr R, Guo W, et al. Induction of pluripotent stem cells by defined factors is greatly improved by small-molecule compounds. Nat Biotechnol. 2008;26:795-7.

59. Obokata H, Wakayama T, Sasai $Y$, et al. Stimulus-triggered fate conversion of somatic cells into pluripotency. Nature. 2014;505:641-7.

60. Hou P, Li Y, Zhang $X$, et al. Pluripotent stem cells induced from mouse somatic cells by small-molecule compounds. Science. 2013;341:651-4.

61. Kim K, Doi A, Wen B, et al. Epigenetic memory in induced pluripotent stem cells. Nature. 2010;467:285-90.

62. Polo JM, Liu S, Figueroa ME, et al. Cell type of origin influences the molecular and functional properties of mouse induced pluripotent stem cells. Nat Biotechnol. 2010;28:848-55.

63. Ohnuki M, Tanabe K, Sutou K, et al. Dynamic regulation of human endogenous retroviruses mediates factor-induced reprogramming and differentiation potential. Proc Natl Acad Sci. 2014;111:12426-31.

64. Rouhani F, Kumasaka N, de Brito MC, et al. Genetic background drives transcriptional variation in human induced pluripotent stem cells. PLoS Genet. 2014;10(6):e1004432.

65. Stadtfeld M, Apostolou E, Akutsu H, et al. Aberrant silencing of imprinted genes on chromosome 12qF1 in mouse induced pluripotent stem cells. Nature. 2010:465:175-81.

66. Lister R, Pelizzola M, Kida YS, et al. Hotspots of aberrant epigenomic reprogramming in human induced pluripotent stem cells. Nature. 2011:471: $68-73$.

67. Zhao T, Zhang Z-N, Rong Z, Xu Y. Immunogenicity of induced pluripotent stem cells. Nature. 2011:474:212-5.

68. Blelloch $\mathrm{R}$, Wang Z, Meissner A, et al. Reprogramming efficiency following somatic cell nuclear transfer is influenced by the differentiation and methylation state of the donor nucleus. Stem Cells. 2006;24:2007-13.

69. Streckfuss-Bömeke K, Wolf F, Azizian A, et al. Comparative study of humaninduced pluripotent stem cells derived from bone marrow cells, hair keratinocytes, and skin fibroblasts. Eur Heart J. 2013:34:2618-29.

70. Nguyen H, Geens M, Spits C. Genetic and epigenetic instability in human pluripotent stem cells. Hum Reprod update. 2013:19(2):187-205.

71. Ma T, Xie M, Laurent T, Ding S. Progress in the reprogramming of somatic cells. Circ Res. 2013:112:562-74.

72. Gura T. Does cloning produce better embryonic stem cells? Science. 2013 340:1390-90.

73. Campbell KH, McWhir J, Ritchie W, Wilmut I. Sheep cloned by nuclear transfer from a cultured cell line. Nature. 1996;380:64-6.

74. Yin XJ, Tani T, Yonemura I, et al. Production of cloned pigs from adult somatic cells by chemically assisted removal of maternal chromosomes. Biol Reprod. 2002;67:442-6.

75. Fulka J, Moor RM. Noninvasive chemical enucleation of mouse oocytes. Mol Reprod Dev. 1993:34:427-30

76. Teperek M, Miyamoto K. Nuclear reprogramming of sperm and somatic nuclei in eggs and oocytes. Reprod Med Biol. 2013;12:133-49.

77. Susko-Parrish J, Leibfried-Rutledge M, Northey D, Schutzkus V, First N. Inhibition of protein kinases after an induced calcium transient causes transition of bovine oocytes to embryonic cycles without meiotic completion. Dev Biol. 1994:166:729-39.

78. Escoffier J, Lee HC, Yassine S, et al. Homozygous mutation of PLCZ1 leads to defective human oocyte activation and infertility that is not rescued by the WW binding protein PAWP. Hum Mol Genet. 2016;25(5):878-91. 
79. Kashir J, Nomikos M, Swann K, Lai FA. PLCל or PAWP: revisiting the putative mammalian sperm factor that triggers egg activation and embryogenesis. Mol Human Reprod. 2015;21(3):383-8.

80. Tesarik J, Sousa M, Testart J. Human oocyte activation after intracytoplasmic sperm injection. Hum Reprod. 1994;9:511-8.

81. Qu P, Wang Y, Zhang C, Liu E. Insights into the roles of sperm in animal cloning. Stem Cell Res Ther. 2020;11(1):1-10.

82. Beaujean N, Taylor JE, McGarry M, et al. The effect of interspecific oocytes on demethylation of sperm DNA. Proc Natl Acad Sci U S A. 2004;101:763640.

83. Kashir J, Nomikos M, Lai FA, Swann K. Sperm-induced Ca 2+ release during egg activation in mammals. Biochem Biophys Res Commun. 2014;450:120411.

84. Morado S, Cetica P, Beconi M, Thompson J, Dalvit G. Reactive oxygen species production and redox state in parthenogenetic and spermmediated bovine oocyte activation. Reproduction. 2013;145:471-8.

85. Jodar M, Selvaraju S, Sendler E, Diamond MP, Krawetz SA. The presence, role and clinical use of spermatozoal RNAs. Hum Reprod Update. 2013;19:60424.

86. Mizutani E, Yamagata K, Ono T, et al. Abnormal chromosome segregation at early cleavage is a major cause of the full-term developmental failure of mouse clones. Dev Biol. 2012;364:56-65.

87. McLay DW, Clarke HJ. Remodelling the paternal chromatin at fertilization in mammals. Reproduction. 2003;125:625-33.

88. Ostermeier GC, Miller D, Huntriss JD, Diamond MP, Krawetz SA Reproductive biology: delivering spermatozoan RNA to the oocyte. Nature. 2004:429:154-54.

89. Miller D, Ostermeier GC. Towards a better understanding of RNA carriage by ejaculate spermatozoa. Hum Reprod Update. 2006;12:757-67.

90. Kumar G, Patel D, Naz R. c-MYC mRNA is present in human sperm cells. Cell Mol Biol Res. 1992;39:111-7.

91. Liu W-M, Pang RT, Chiu PC, et al. Sperm-borne microRNA-34c is required for the first cleavage division in mouse. Proc Natl Acad Sci. 2012;109:490-4.

92. Hammoud SS, Nix DA, Zhang $\mathrm{H}$, et al. Distinctive chromatin in human sperm packages genes for embryo development. Nature. 2009;460:473-8.

93. Kim K, Zhao R, Doi A, et al. Donor cell type can influence the epigenome and differentiation potential of human induced pluripotent stem cells. Nat Biotechnol. 2011:29:1117-9.

94. Chin MH, Mason MJ, Xie W, et al. Induced pluripotent stem cells and embryonic stem cells are distinguished by gene expression signatures. Cell Stem Cell. 2009:5:111-23

95. Ghosh Z, Wilson KD, Wu Y, et al. Persistent donor cell gene expression among human induced pluripotent stem cells contributes to differences with human embryonic stem cells. PLoS One. 2010;5:e8975.

96. Ohi Y, Qin H, Hong C, et al. Incomplete DNA methylation underlies a transcriptional memory of somatic cells in human iPS cells. Nat Cell Biol. 2011;13:541-9.

97. Greaves LC, Turnbull DM. Mitochondrial DNA mutations and ageing Biochimica et Biophysica Acta (BBA)-General Subjects. 2009:1790:1015-20.

98. Reeve AK, Krishnan KJ, Turnbull D. Mitochondrial DNA mutations in disease, aging, and neurodegeneration. Ann N Y Acad Sci. 2008;1147:21-9.

99. Santos C, Martinez M, Lima M, et al. Mitochondrial DNA mutations in cancer: a review. Curr Top Med Chem. 2008;8:1351-66.

100. Prigione A, Hossini AM, Lichtner B, et al. Mitochondrial-associated cell death mechanisms are reset to an embryonic-like state in aged donor-derived iPS cells harboring chromosomal aberrations. PLoS One. 2011;6:e27352.

101. Greggains GD, Lister LM, Tuppen HA, et al. Therapeutic potential of somatic cell nuclear transfer for degenerative disease caused by mitochondrial DNA mutations. Sci Rep. 2014;4:1-10.

102. Wai T, Ao A, Zhang $X$, et al. The role of mitochondrial DNA copy number in mammalian fertility. Biol Reprod. 2010;83:52-62.

103. Danan C, Sternberg D, Van Steirteghem A, et al. Evaluation of parental mitochondrial inheritance in neonates born after intracytoplasmic sperm injection. Am J Hum Genet. 1999;65:463-73.

104. Ogura A, Inoue K, Wakayama T. Recent advancements in cloning by somatic cell nuclear transfer. Philos Trans Royal Soc B: Biol Sci. 2013:368:20110329.

105. Blackburn EH, Epel ES, Lin J. Human telomere biology: a contributory and interactive factor in aging, disease risks, and protection. Science. 2015;350: 1193-8.

106. Flores I, Blasco MA. The role of telomeres and telomerase in stem cell aging. FEBS Lett. 2010;584:3826-30.
107. Huang J, Wang F, Okuka M, et al. Association of telomere length with authentic pluripotency of ES/iPS cells. Cell Res. 2011;21:779-92.

108. Marión RM, Strati K, Li H, et al. A p53-mediated DNA damage response limits reprogramming to ensure iPS cell genomic integrity. Nature. 2009;460: 1149-53.

109. Jeon $H$, Hyun $\mathrm{S}$, Lee $\mathrm{G}$, et al. The analysis of telomere length and telomerase activity in cloned pigs and cows. Mol Reprod Dev. 2005;71:31520.

110. Betts DH, Bordignon V, Hill JR, et al. Reprogramming of telomerase activity and rebuilding of telomere length in cloned cattle. Proc Natl Acad Sci. 2001; 98:1077-82.

111. Marion RM, Strati K, Li H, et al. Telomeres acquire embryonic stem cell characteristics in induced pluripotent stem cells. Cell Stem Cell. 2009;4:14154

112. Le $R$, Kou $Z$, Jiang $Y$, et al. Enhanced telomere rejuvenation in pluripotent cells reprogrammed via nuclear transfer relative to induced pluripotent stem cells. Cell Stem Cell. 2014;14:27-39.

113. Ozturk S. Telomerase activity and telomere length in male germ cells. Biol Reprod. 2015;92:53.

114. Shuai L, Zhou Q. Haploid embryonic stem cells serve as a new tool for mammalian genetic study. Stem Cell Res Ther. 2014;5:20.

115. Elling U, Woods M, Forment JV, et al. Derivation and maintenance of mouse haploid embryonic stem cells. Nat Protoc. 2019;14:1991-2014.

116. Yang $H$, Shi L, Wang B-A, et al. Generation of genetically modified mice by oocyte injection of androgenetic haploid embryonic stem cells. Cell. 2012; 149:605-17.

117. Li W, Shuai L, Wan H, et al. Androgenetic haploid embryonic stem cells produce live transgenic mice. Nature. 2012;490:407-11.

118. Ding C, Huang S, Qi Q, et al. Derivation of a homozygous human androgenetic embryonic stem cell line. Stem Cells Dev. 2015;24:2307-16.

119. Wang X, Miller DC, Harman R, Antczak DF, Clark AG. Paternally expressed genes predominate in the placenta. Proc Natl Acad Sci. 2013;110:10705-10.

120. Kono T, Sotomaru Y, Sato Y, Nakahara T. Development of androgenetic mouse embryos produced by in vitro fertilization of enucleated oocytes. Mol Reprod Dev. 1993;34:43-6.

121. Lagutina I, Lazzari G, Duchi R, Galli C. Developmental potential of bovine androgenetic and parthenogenetic embryos: a comparative study. Biol Reprod. 2004;70:400-5.

122. Matsukawa K, Turco MY, Scapolo PA, et al. Development of sheep androgenetic embryos is boosted following transfer of male pronuclei into androgenetic hemizygotes. Cloning Stem Cells. 2007;9:374-81.

123. Latham KE, Akutsu H, Patel B, Yanagimachi R. Comparison of gene expression during preimplantation development between diploid and haploid mouse embryos. Biol Reprod. 2002;67:386-92.

124. Teramura T, Onodera Y, Murakami H, et al. Mouse androgenetic embryonic stem cells differentiated to multiple cell lineages in three embryonic germ layers in vitro. J Reprod Dev. 2009:55:283-92.

125. Dinger TC, Eckardt S, Choi SW, et al. Androgenetic embryonic stem cells form neural progenitor cells in vivo and in vitro. Stem Cells. 2008;26:147483.

126. Hayakawa T, Aoi T, Umezawa A, et al. A study on ensuring the quality and safety of pharmaceuticals and medical devices derived from the processing of human embryonic stem cells. Regen Ther. 2015:2:109-22.

127. Gore A, Li Z, Fung H-L, et al. Somatic coding mutations in human induced pluripotent stem cells. Nature. 2011:471:63-7.

128. Wells D, Forsyth J, McMillan V, Oback B. Review: the health of somatic cell cloned cattle and their offspring. Cloning Stem Cells. 2004;6:101-10.

129. Elbracht M, Mackay D, Begemann M, Kagan KO, Eggermann T. Disturbed genomic imprinting and its relevance for human reproduction: causes and clinical consequences. Hum Reprod Update. 2020;26:197-213.

130. Li E, Beard C, Jaenisch R. Role for DNA methylation in genomic imprinting. Nature. 1993:366:362-5.

131. Ortega-Recalde $O$, Hore TA. DNA methylation in the vertebrate germline: balancing memory and erasure. Essays Biochem. 2019;63:649-61.

132. Neumann B, Kubicka P, Barlow DP. Characteristics of imprinted genes. Nat Genet. 1995:9:12-3.

133. Amor DJ, Halliday J. A review of known imprinting syndromes and their association with assisted reproduction technologies. Hum Reprod. 2008;23 2826-34.

134. Reik W, Walter J. Genomic imprinting: parental influence on the genome Nat Rev Genet. 2001;2:21-32. 
135. Reik W, Dean W, Walter J. Epigenetic reprogramming in mammalian development. Science. 2001;293:1089-93.

136. Shen C-J, Lin C-C, Shen P-C, et al. Imprinted genes and satellite loci are differentially methylated in bovine somatic cell nuclear transfer clones. Cellular Reprogramming (Formerly "Cloning and Stem Cells"). 2013;15:41324.

137. Chang G, Liu S, Wang F, et al. Differential methylation status of imprinted genes in nuclear transfer derived ES (NT-ES) cells. Genomics. 2009;93:112-9.

138. Arnold DR, Gaspar RC, da Rocha CV, et al. Nuclear transfer alters placental gene expression and associated histone modifications of the placentalspecific imprinted gene pleckstrin homology-like domain, family A, member 2 (PHLDA2) in cattle. Reprod Fertil Dev. 2017;29(3):458-67.

139. Bressan F, Therrien J, Filion F, et al. 331 abnormal DNA methylation patterns and allele-specific expression of imprinted genes in bovine-induced pluripotent stem cells. Reprod Fertil Dev 2015:27:254-54.

140. Tiemann U, Wu G, Marthaler AG, Schöler HR, Tapia N. Epigenetic aberrations are not specific to transcription factor-mediated reprogramming. Stem Cell Rep. 2016;6:35-43.

141. Tucci V, Isles AR, Kelsey G, et al. Genomic imprinting and physiological processes in mammals. Cell. 2019;176:952-65.

142. Miyamoto K. Various nuclear reprogramming systems using egg and oocyte materials. J Reprod Develop. 2019:65(3):203-8.

143. Poirier M, Smith OE, Therrien J, et al. Resiliency of equid H19 imprint to somatic cell reprogramming by oocyte nuclear transfer and genetically induced pluripotencyt. Biol Reprod. 2019;102:211-9.

144. Payer B, Lee JT. X chromosome dosage compensation: how mammals keep the balance. Annu Rev Genet. 2008:42:733-72.

145. Xue F, Tian XC, Du F, et al. Aberrant patterns of X chromosome inactivation in bovine clones. Nat Genet. 2002;31:216-20.

146. Tchieu J, Kuoy E, Chin MH, et al. Female human iPSCs retain an inactive $X$ chromosome. Cell Stem Cell. 2010;7:329-42.

147. Lessing DMC, Lee JT. $X$ chromosome inactivation and epigenetic responses to cellular reprogramming. Annu Rev Genomics Hum Genet. 2013;14:85110.

148. Sebban S, Buganim Y. Nuclear reprogramming by defined factors: quantity versus quality. Trends Cell Biol. 2016;26:65-75.

149. Li Z, Lu H, Yang W, et al. Mouse SCNT ESCs have lower somatic mutation load than syngeneic iPSCs. Stem Cell Rep. 2014;2:399-405.

150. Okano H, Nakamura M, Yoshida K, et al. Steps toward safe cell therapy using induced pluripotent stem cells. Circ Res. 2013;112:523-33.

151. Hu C, Li L. Current reprogramming systems in regenerative medicine: from somatic cells to induced pluripotent stem cells. Regen Med. 2016;11:105-32.

\section{Publisher's Note}

Springer Nature remains neutral with regard to jurisdictional claims in published maps and institutional affiliations. 